\title{
Polarons radical polymerization
}

\author{
Kohei Yamabe, Kuniharu Nakajima, Hiromasa Goto* \\ Department of Materials Science, Faculty of Pure and Applied Sciences, University of Tsukuba, \\ Tsukuba, Ibaraki 305-8573 Japan \\ Correspondence to: Hiromasa Goto (E-mail:gotoh@ims.tsukuba.ac.jp)
}

\begin{abstract}
Polystyrene has been typically prepared with radical polymerization by benzoyl peroxide (BPO) or azobisisobutyronitrile (AIBN). In this report, polymerization of styrene was carried out by radical cations of polyaniline (PANI). Polarons of conducting polymers are consisting of radical cations. The polarons bear electrical conduction as a charge carrier. We employ the polarons as an initiator for radical polymerization. Polymerization of styrene and acrylonitrile by the polarons was conducted to explore new possibility of conducting polymers. Fourier-transfer infrared absorption (FT-IR) spectroscopy measurements for the resultant polymers obtained with polarons of polyaniline indicates that the polystyrene thus synthesized grows from polyaniline. The qualitative solubility, average molecular weight, and thermal stability are comparable to that of polystyrene obtained by the common method with BPO. Radical polymerization by polarons may provide a new avenue for radical polymerizations through application of conducting polymer.
\end{abstract}

KEYWORDS: initiator; polyaniline; polarons; polystyrene; radical polymerization.

\section{INTRODUCTION}

B enzoyl peroxide (BPO) is commonly used as an initiator in radical polymerization to produce polymeric materials such as polystylene. ${ }^{1-3}$ BPO generates radical species with heat treatment. The radical initiates chain reaction for polymerization. Molecular weights and synthetic yields of the product depend on concentration of the initiator, reaction time, and reaction temperature. ${ }^{1,2}$ Large amount of the initiator in the polymerization depresses increase of molecular weight of the resultant polymers due to bi-molecular termination. ${ }^{2}$

Polyaniline (PANI) is a historical polymer in the field of conducting polymer studies. Many researches on PANI have been carried out in both fundamental and application viewpoints because of low cost, thermal stability, and environmental stability. Tuning of doping level (oxidation level) allows change in physical properties of PANI such as conductivity. PANI consists of various molecular structure of combination of benzenoid and quinoid sequence with 
polarons (radical cations) on the nitrogen atom. Especially, emeraldine salt of PANI shows good electrical conductivity. ${ }^{4,5}$ Electrical conductivity of the polymers comes from polarons in the form of radical cations on the conjugated skeleton generated by doping. ${ }^{6}$

In this study, polymerization of styrene and acrylonitrile by polarons on conducting polymer as a polymerization initiator is carried out. An application of polarons as a charge carrier for polymerization can be a first example in polymer chemistry. The method proposed in this study demonstrates a new function of charge carriers for obtaining plastics.

\section{EXPERIMENTAL}

Materials. Dimethyl sulfoxide (DMSO) and 4-bromo-2,6-di-tert-butylphenol were purchased from Wako Chemical, Japan, and used as received. Styrene and acrylonitrile were purchased from Tokyo Chemical Industry (TCI), Japan, and purified by distillation. Ammonium persulfate (APS, $\left.\left(\mathrm{NH}_{4}\right)_{2} \mathrm{~S}_{2} \mathrm{O}_{8}\right)$ was purchased from Yoneyama Chemical, Japan, and used as received. Nmethylpyrrolidone (NMP), tetrahydrofuran (THF), chloroform, hexane, and methanol were purchased from Nacalai tesque, Japan, and used as received. PANI was previously synthesized in the distilled water in the presence of APS and sulfuric acid at $\sim 0^{\circ} \mathrm{C}$ as general method. Preparation and characterization detail of the PANI is described in Supporting Information (SI).

Instruments. Infrared (IR) absorption spectra for the polymer samples were obtained with a FT/IR-4600 spectrometer (Jasco, Japan) by the $\mathrm{KBr}$ method. UV-vis absorption spectra were measured with a V-630 UV-vis optical absorption spectrometer (Jasco, Japan). Thermogravimetric analysis for polymer samples were carried out with an EXSTAR7000 (Seiko, Japan). Molecular weights of the samples were obtained with a MD915 and PI980 (Jasco, Japan) against polystyrene standard. Unpaired electrons in the polymer were detected with an EMX-T ESR Spectrometer (Bruker, USA).

Polymerization by polarons. PANI was completely dissolved in NMP with vigorously stirring under an argon atmosphere. Then, styrene (monomer) was added to the PANI solution. The reaction mixture was heated at $75{ }^{\circ} \mathrm{C}$ to initiate polymerization. After $24 \mathrm{~h}$, minimal amount of THF was added to dissolve the polymer. The polymer solution in THF was slowly dropped into large volume of methanol. A pale pink precipitant appeared in the solution. In order to remove

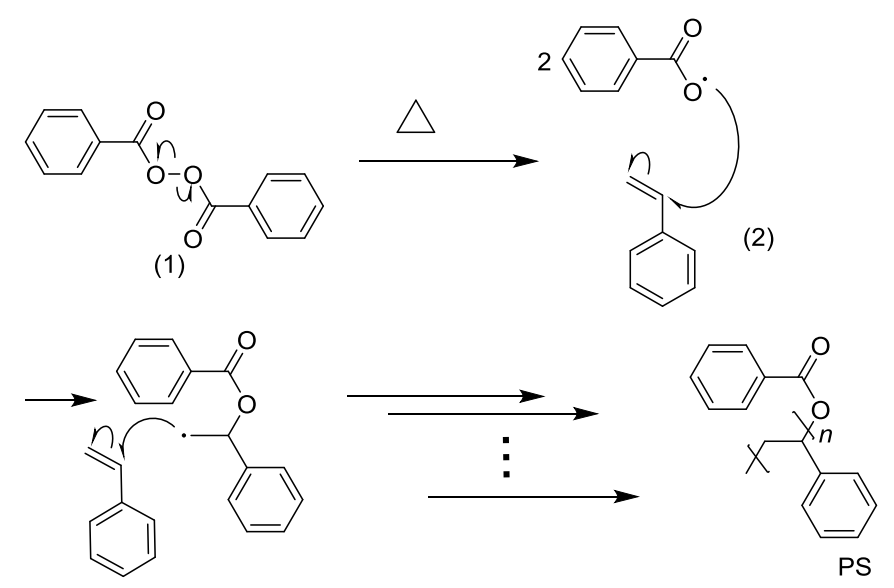

SCHEME 1 Polymerization mechanism of styrene initiated with BPO. oligomers and residual NMP, the precipitant was washed by methanol and distilled water in several times. After wash and filtration, the solid was dried in vacuum to evaporate solvent in the sample completely.

A reference PS was prepared with BPO in NMP. Polymerizations with the PANI in DMSO were also carried out. The polymerization condition and the results are summarized in Table 1. Extension of the reaction time increases synthetic yield. The polymers thus obtained are 
abbreviated as PS (polystyrene), PANI, PS-PANI $n / \mathrm{ES} m$ ( $n=$ additional amount of PANI in the polymerization $(\mathrm{mg}), \mathrm{ES}=$ emeraldine salt, $m=$ PANI sample 1 or 2$)$, PS-PANI-D/ES1 $(\mathrm{D}=$ DMSO as polymerization solvent), and PS-PANI72h/ES1 (72 $\mathrm{h}=$ reaction time), SI (Supporting Information). Polymerizations with the reduced PANI as emeraldine base (half-dedoping form) having low amount of radical species is also examined. However, the reduced form produced trace amount polymers because of low radical concentration (PS-PANI5/EB, PS-PANI10/EB, PS-PANI30/EB), Table 2. Furthermore, experimental investigation for reaction temperature in the polymerization is shown in Table S1 (Supporting Information). Polymerization mechanism of styrene initiated with BPO is described in Scheme 1. First, dimerization by heating is occurred to produce two oxy radicals (Scheme 1(1)). Next, the radical on the oxygen attacks to double bond of styrene to initiate polymerization reaction (Scheme 1(2)). The terminal carbon radical on the styrene unit further attack to styrene as a monomer. The chain reaction constructs polystyrene (PS) having great molecular weight from BPO in the terminal. In the same way, radicals of PANI polarons attack to styrene as a monomer (Scheme 2(1,2). Further chain reaction allows production of high molecular weight PS having PANI in the terminal, Scheme 2(3,4). A doped form of PANI (emeraldine salt, ES) has several numbers of polarons unit in the main chain.

TABLE 1 Preparation of polystyrene (PS) ${ }^{\mathrm{a}}$.

\begin{tabular}{|c|c|c|c|c|c|c|}
\hline \multirow[t]{2}{*}{ Polymer ${ }^{b}$} & \multicolumn{2}{|c|}{ Initiator $^{\mathrm{c}}$} & \multirow{2}{*}{$\begin{array}{c}\text { Time }^{f} \\
\text { (h) }\end{array}$} & \multirow{2}{*}{$\begin{array}{c}\text { Solvent }^{\mathrm{g}} \\
(2.0 \\
\mathrm{mL})\end{array}$} & \multicolumn{2}{|c|}{ Yield } \\
\hline & PANI-ES1 ${ }^{\mathrm{d}}(\mathrm{mg})$ & $\mathrm{BPO}^{\mathrm{e}}(\mathrm{mg})$ & & & (quantity, g) & $\%$ \\
\hline $\mathrm{PS}^{\mathrm{h}}$ & - & 20 & 24 & $\mathrm{NMP}^{\mathrm{i}}$ & 3.6 & 99 \\
\hline PS-PANI0 & 0 & - & 24 & $\mathrm{NMP}^{\mathrm{i}}$ & - & - \\
\hline PS-PANI3/ES1 & 3.0 & - & 24 & $\mathrm{NMP}^{\mathrm{i}}$ & 0.54 & 15 \\
\hline PS-PANI5/ES1 & 5.0 & - & 24 & $\mathrm{NMP}^{\mathrm{i}}$ & 0.61 & 17 \\
\hline PS-PANI10/ES1 & 10 & - & 24 & $\mathrm{NMP}^{\mathrm{i}}$ & 0.38 & 11 \\
\hline PS-PANI30/ES1 & 30 & - & 24 & $\mathrm{NMP}^{\mathrm{i}}$ & - & - \\
\hline PS-PANI60/ES1 & 60 & - & 24 & $\mathrm{NMP}^{\mathrm{i}}$ & - & - \\
\hline PS-PANI-D/ES1 & 5.0 & - & 24 & $\mathrm{DMSO}^{\mathrm{j}}$ & 0.35 & 9.7 \\
\hline PS-PANI3-2/ES1 & 3.0 & - & 72 & $\mathrm{NMP}^{1}$ & 0.85 & 24 \\
\hline \multicolumn{7}{|c|}{ 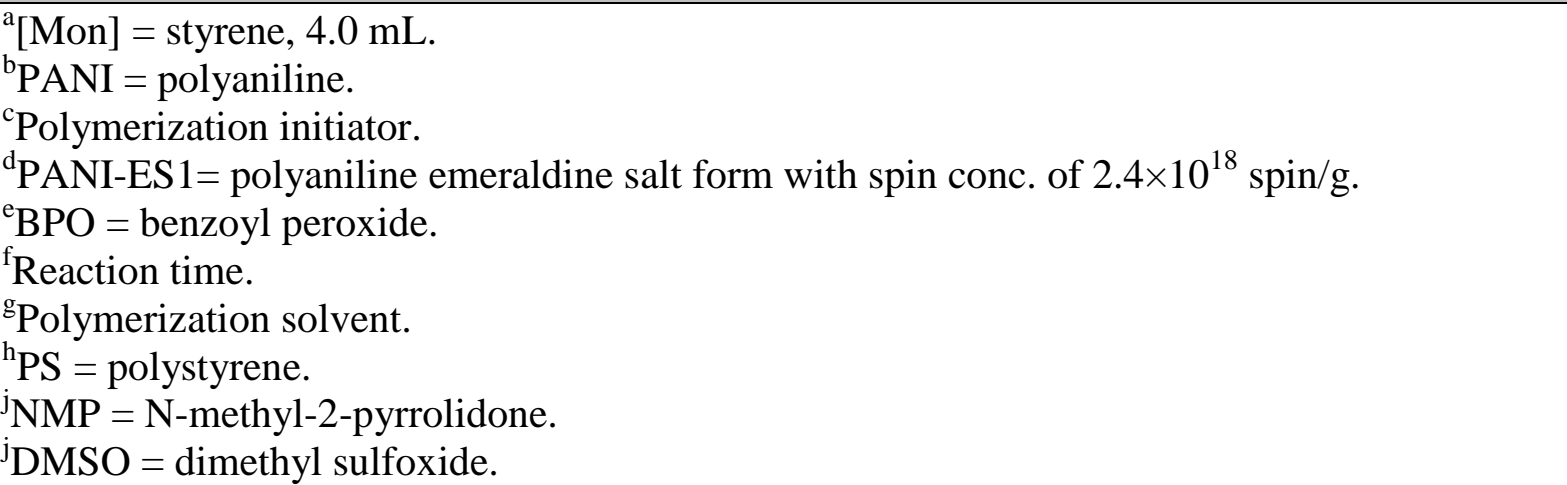 } \\
\hline
\end{tabular}



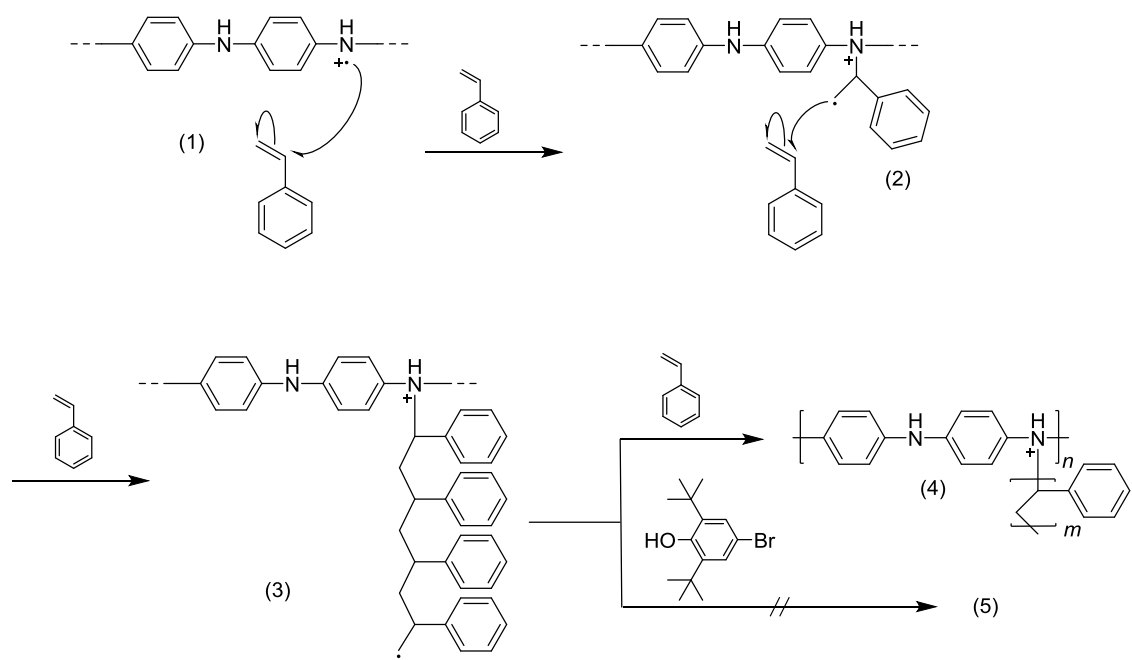

SCHEME 2 Polymerization mechanism of styrene initiated with PANI polarons.
Possible chemical structure of the PS is shown in Figure 1. The plural number polarons can initiate the polymerization to allow random arranged form because PS grows from plural polarons site in 3D directions from individual main chain of PANI, as shown in inset of Figure 1. Copolymerization of styrene and acrylonitrile are carried out with the PANI polarons (Scheme 3). The copolymers thus obtained are abbreviate as $\mathrm{P}(\mathrm{St}-\mathrm{co}-\mathrm{ACN})-\mathrm{PANI} / \mathrm{ES} 1$. A radical scavenger compound (4-bromo2,6-di-tert-butylphenol) was employed to capture of the radicals in the polymerization to consider function of the PANI polarons in the polymerization. ${ }^{7}$ The radical scavenger can depress activity of polymerization initiator of PANI polarons, resulting yield of PS-PANI/ES1 thus obtained was quite low, Table 3.

TABLE 2 Preparation of polystyrene (PS) by polyaniline (PANI) emeraldine base (low spin conc.) and emeraldine salt (high spin conc.) ${ }^{\mathrm{a}}$.

\begin{tabular}{cccccc}
\hline Polymer & \multicolumn{2}{c}{ Initiator $(\mathrm{mg})^{2}$} & \multicolumn{2}{c}{ NMP $^{\mathrm{d}}$} & \multicolumn{2}{c}{ Yield $^{(\mathrm{mL})}$} & $(\mathrm{g})$ & $\%$ \\
\hline PANI-EB $^{\mathrm{b}}$ & PANI-ES2 $^{\mathrm{c}}$ & & 2.0 & - & - \\
PS-PANI5/EB $^{\mathrm{b}}$ & 5.0 & - & 2.0 & - & - \\
PS-PANI10/EB $^{\mathrm{b}}$ & 10 & - & 2.0 & - & - \\
\hline PS-PANI5/ES2 $^{\mathrm{c}}$ & 30 & - & 2.0 & 1.6 & 44 \\
PS-PANI15/ES2 $^{\mathrm{c}}$ & - & 5.0 & 2.0 & 1.2 & 33 \\
PS-PANI30/ES2 $^{\mathrm{c}}$ & - & 15 & 2.0 & 1.0 & 28 \\
PS-PANI60/ES2 $^{\mathrm{c}}$ & - & 30 & 2.0 & - & - \\
\hline
\end{tabular}

\footnotetext{
${ }^{\mathrm{a}}$ Mon. = styrene, $4.0 \mathrm{~mL}$.

${ }^{\mathrm{b}} \mathrm{EB}=$ emeraldine base (reduced form), spin conc. $=1.8 \times 10^{15} \mathrm{spin} / \mathrm{g}$.

${ }^{\mathrm{c}} \mathrm{ES} 2=$ emeraldine salt $\left(\right.$ oxidized form), spin conc. $=3.8 \times 10^{19} \mathrm{spin} / \mathrm{g}$.

${ }^{\mathrm{d}} \mathrm{NMP}=\mathrm{N}$-methyl-2-pyrrolidone (polymerization solvent).
} 


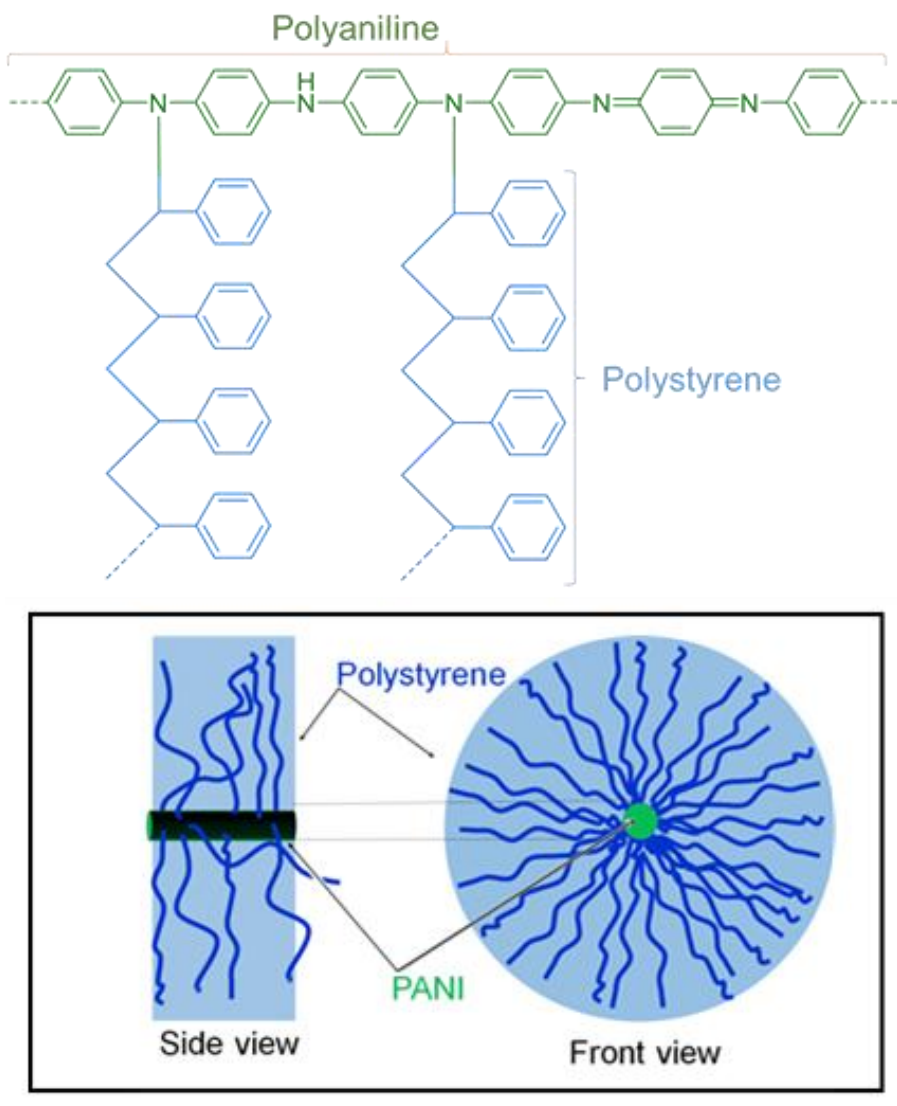

The radical scavenger depressed polymerization reaction.Termination of the polymerization by the radical scavenger confirms no possibility of cationic polymerization process with positive charge of the polarons, Scheme 2(5). Synthesis of poly(styrene-co-acrylonitrile) was successfully carried out with the PANI polarons. Copolymerization of styreneacrylonitrile is known for well progressing reaction in radical polymerization process. These results conclude that the polymerization by polarons of PANI proceeds in radical polymerization process. PS chains may grow from PANI compact coil form or expanded coil. $^{7}$

FIGURE 1 Molecular form of polystyrene prepared with polyaniline. (Inset): Possible molecular form of the polymer prepared with polarons of polyaniline (PANI).

TABLE 3 Preparation of poly(styrene-co-acrylonitrile) ${ }^{\mathrm{a}}$ and polymerization in the presence of a radical scavenger ${ }^{\mathrm{a}}$.

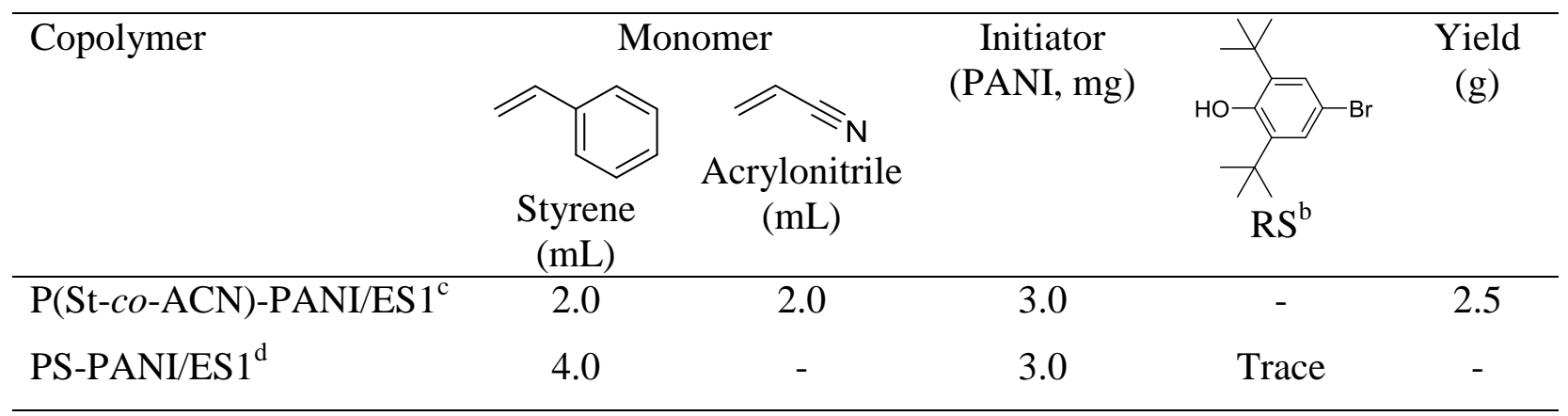

\footnotetext{
salt form. Spin conc. $\left.=2.4 \times 10^{18} \mathrm{spin} / \mathrm{g}\right)$.

${ }^{\mathrm{b}} \mathrm{RS}=$ radical scavenger (4-bromo-2,6-di-tert-butylphenol).

${ }^{\mathrm{c}}$ Poly(styrene-co-acrylonitrile) prepared with PANI emeraldine salt.

${ }^{\mathrm{d}}$ Polystyrene prepared with PANI emeraldine salt.
}

${ }^{a}$ Polymerization in $2.0 \mathrm{~mL}$ of N-methyl-2-pyrrolidone (NMP) with ES1 (polyaniline emeraldine 


\section{RESULTS AND DISCUSSION}

Characterization. FT-IR measurements with $\mathrm{KBr}$ method were carried out to identify chemical structure of the polymers. IR spectra of PANI, PS-PANI3/ES1, PS-PANI5/ES1, PS-PANI10/ES1, PS-PANI-D/ES1, and PS of 400-4000 $\mathrm{cm}^{-1}$ and $1000-1500 \mathrm{~cm}^{-1}$ regions are shown in Figure 2. Absorptions of aromatic $\mathrm{C}-\mathrm{H}$ stretching at around $3050 \mathrm{~cm}^{-1}$, alkyl C-H stretching of PS at around $2900 \mathrm{~cm}^{-1}$, aromatic $\mathrm{C}-\mathrm{C}$ stretching at around $1470 \mathrm{~cm}^{-1}, \mathrm{C}-\mathrm{H}$ out-of-plane bending at $765 \mathrm{~cm}^{-1}$, and aromatic $\mathrm{C}-\mathrm{C}$ out-of-plane bending at $700 \mathrm{~cm}^{-1}$ are identical as polystyrene. ${ }^{1}$ This result confirms that the resultant polymers prepared by the polarons are to be polystyrene, indicating polarons initiate polymerization for the unsaturated monomer. PANI shows absorption of the C-N stretching in the range of $1100-1400 \mathrm{~cm}^{-1}$. The absorption bands of $1380,1315,1240$ $\mathrm{cm}^{-1}$ are assigned to aromatic amine of PANI. ${ }^{9}$ PS-PANI samples show absorption band at 1262 $\mathrm{cm}^{-1}$. According to previous report, N-alkyl PANI show an absorption band at $1252 \mathrm{~cm}^{-1}$ band derived from $\mathrm{C}-\mathrm{N}$ stretching of the alkyl substituents linked on the amine nitrogen. ${ }^{10-12} \mathrm{An}$ intense absorption ( $\mathrm{C}=\mathrm{O}$ stretching) at $1690 \mathrm{~cm}^{-1}$ due to NMP is observed, indicating the samples still contain small amount of residual NMP. ${ }^{13}$ PS-PANI-D/ES1 (prepared in DMSO) also shows absorption band at $1262 \mathrm{~cm}^{-1}$, Figure 2 right. In previous report, aromatic ring-substituted PANI showed new absorption bands assigned to aromatic C-H in $1100-800 \mathrm{~cm}^{-1}$ regions ${ }^{14,15}$. However, no new absorption bands in $1100-800 \mathrm{~cm}^{-1}$ region were observed in the all PS-PANI samples, indicating occurance of formation of linkage between PANI and PS at N-position.The absorption band of $1262 \mathrm{~cm}^{-1}$ is due to $\mathrm{C}-\mathrm{N}$ bonds between $\mathrm{N}$-position of PANI and the terminal of PS. These results indicate that styrene was successfully polymerized by polarons of the PANI with radical polymerization mechanism.
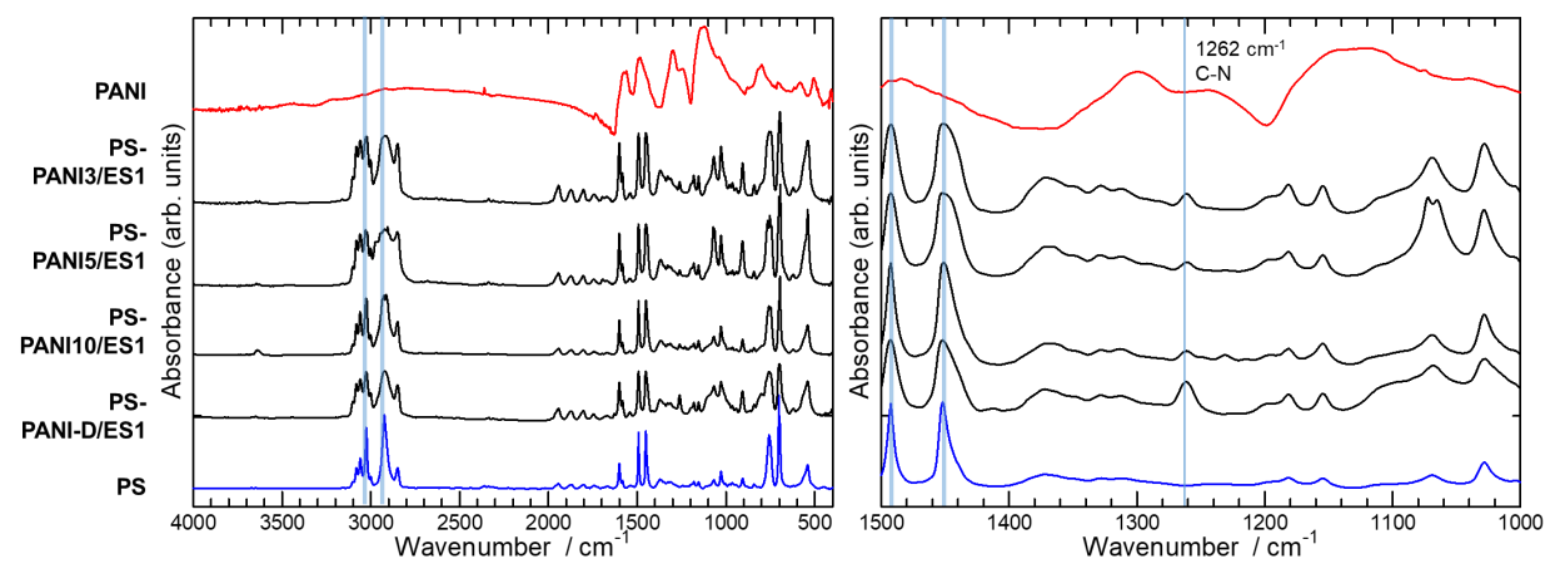

FIGURE 2 FT-IR spectra of polystyrene prepared with benzoyl peroxide and polyaniline (PANI) as initiators, PS, PANI, PS-PANI3/ES1, PS-PANI5/ES1, PS-PANI10/ES1, PSPANI30/ES1, PS-PANI60/ES1, PS-PANI-D/ES1, and PS-PANI3-2/ES1. PS = polystyrene, PANI $n=$ polyaniline and $n=$ additional amount of PANI in the polymerization $(\mathrm{mg}), \mathrm{ES} 1=$ polyaniline emeraldine salt form with spin conc. of $2.4 \times 10^{18} \mathrm{spin} / \mathrm{g}$. D = DMSO (dimethyl sulfoxide) used in the polymerization. (Left): Absorption band from 400-4000 $\mathrm{cm}^{-1}$. (Right): Absorptions at short wavelengths. 
PS-PANI5/ES2 and PS-PANI15/ES2 show the same signals as those of PS-PANIn/ES1, Figure 3. They also exhibit an absorption band at $1262 \mathrm{~cm}^{-1}$, as shown in Figure 3(top right). The copolymer consisting of PS and polyacrylonitrile, abbreviated as P(St-co-ACN)-PANI/ES1, shows the absorptions derived from PS, PAN and PANI, Figure 3(bottom). Absorptions at around $3050 \mathrm{~cm}^{-1}, 2900 \mathrm{~cm}^{-1}, 1470 \mathrm{~cm}^{-1}, 765 \mathrm{~cm}^{-1}$, and $700 \mathrm{~cm}^{-1}$ are identical as polystyrene. ${ }^{1}$ Characteristic absorption bands at 2940 and $2240 \mathrm{~cm}^{-1}$ are respectively assigned to absorption of $\mathrm{C}-\mathrm{H}$ and $\mathrm{C} \equiv \mathrm{N}$ of PAN. ${ }^{16}$ The copolymer shows absorption band at $1259 \mathrm{~cm}^{-1}$, Figure 3 (bottom right). This band may overlap two bands of $1254 \mathrm{~cm}^{-1}\left(\mathrm{C}-\mathrm{N}\right.$ of PAN) and $1262 \mathrm{~cm}^{-1}(\mathrm{C}-\mathrm{N}$ between PANI and copolymer). The spectra show absorption due to chemical structure of PS, PAN and $\mathrm{C}-\mathrm{N}$ bond between PANI and the copolymer. This result indicates that the copolymerization to produce poly(styrene-co-acrylonitrile) was successfully carried out with PANI polarons.
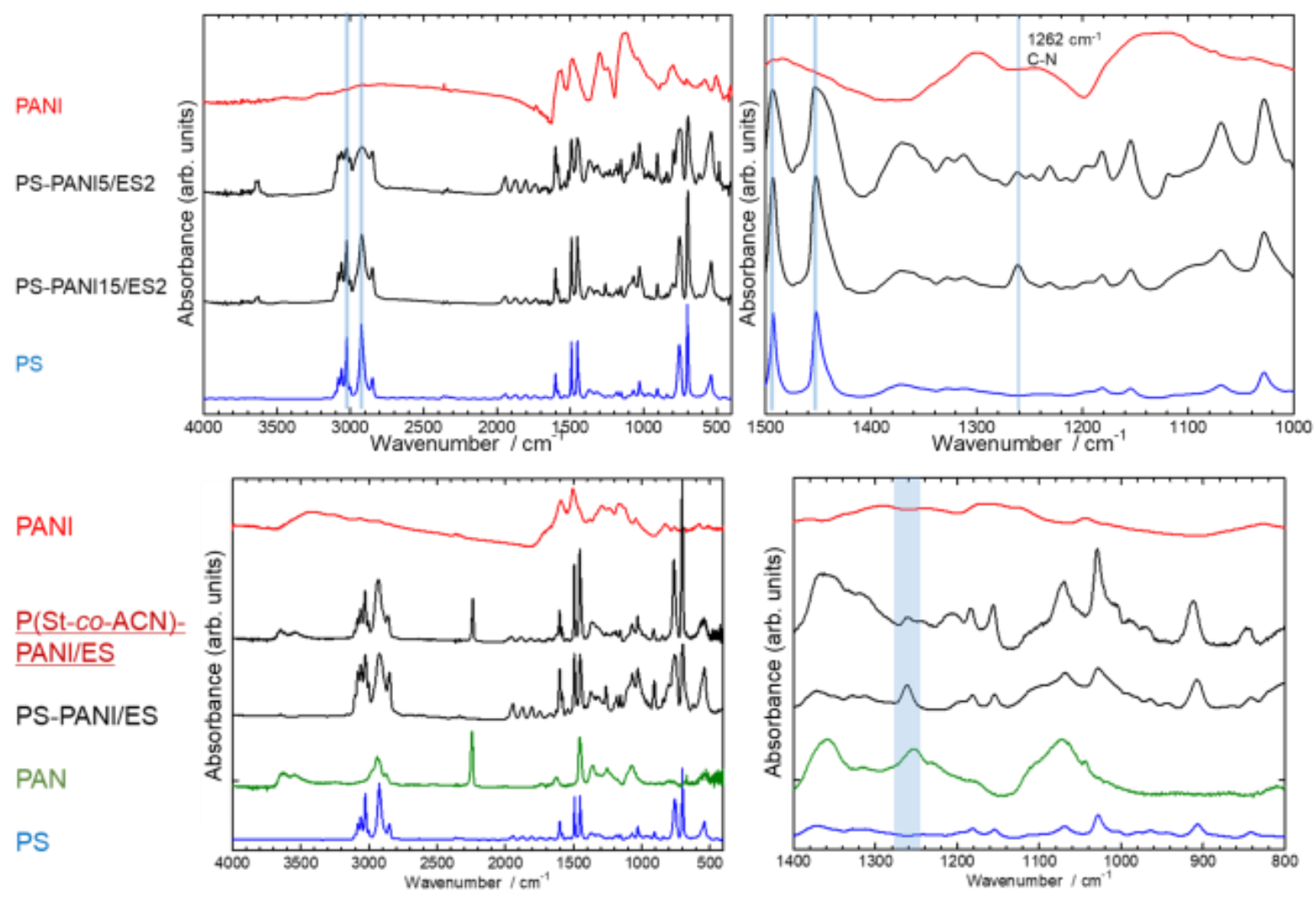

FIGURE 3 FT-IR spectra of polystyrene prepared with benzoyl peroxide and polyaniline (PANI) as initiators, PS, PANI, PS-PANI5/ES2, PS- PANI15/ES2, P(St-co-ACN)-PANI/ES1, and polyacrylonitryle $(\mathrm{PAN})$. PS $=$ polystyrene, $\mathrm{PANI} n=$ polyaniline and $n=$ additional amount of PANI in the polymerization (mg), ES2 = polyaniline emeraldine salt form with spin conc. of $3.8 \times 10^{19} \mathrm{spin} / \mathrm{g}$. (Left): Absorption band from 400-4000 $\mathrm{cm}^{-1}$. (Right): Absorptions at short wavelengths.

Polymer property. Solubility of the polymers is summarized in Table 4. The polymers thus synthesized with PANI polarons have good solubility in common organic solvents. Although pure PANI is dissolved in NMP and DMSO very well, PS and PS-PANI show poor solubility in DMSO. The molecular weights of the polymers were evaluated with gel permeation 
chromatography (GPC) against commercially obtained PS standard, Table 5. The molecular weights of the polymers are in the range of $\sim 10^{5} \mathrm{~g} / \mathrm{mol}$. Appropriate concentration of initiator and polymerization reaction can produce the polymer having viscosity-average molecular weight $\left(M_{\mathrm{v}}\right)$ of $3.5 \times 10^{4} \mathrm{~g} / \mathrm{mol}^{1,2}$ The samples prepared in this study achieved comparable $M_{\mathrm{v}}$ value with the good condition in the common method with BPO or AIBN. Generally, the molecular weight of PS is depended on initiator concentration. ${ }^{1}$ The termination rate is proportional to the concentration of radical species. If the termination occurs before well growth of the polymer chain, molecular weight of polymer is depressed. High concentration of PANI to styrene results in low molecular weight because of occurrence of bi-radical termination reaction such as dismutation and recombination. The GPC results evaluate that an appropriate ratio of the polarons of PANI as an initiator against styrene monomer is to be $0.3-0.1 \mathrm{wt} \%$.

TABLE 4 Qualitative solubility of polymers ${ }^{\mathrm{a}}$.

\begin{tabular}{|c|c|c|c|c|c|c|c|}
\hline Polymer $^{b, c}$ & $\mathrm{DMSO}^{\mathrm{d}}$ & $\mathrm{NMP}^{\mathrm{e}}$ & $\mathrm{CHCl}_{3}$ & $\mathrm{THF}^{\mathrm{f}}$ & Toluene & Hexane & Dioxane \\
\hline PS & - & ++ & ++ & ++ & ++ & ++ & + \\
\hline PANI & ++ & ++ & - & - & - & - & - \\
\hline PS-PANI3/ES $1^{\mathrm{f}}$ & - & ++ & ++ & ++ & ++ & + & + \\
\hline PS-PANI5/ES $1^{\mathrm{f}}$ & - & ++ & ++ & ++ & ++ & + & + \\
\hline PS-PANI10/ES1 ${ }^{\mathrm{f}}$ & - & ++ & ++ & ++ & ++ & + & + \\
\hline
\end{tabular}

${ }^{\mathrm{a}}++$, well soluble; + , partially soluble; -, slightly soluble or insoluble.

${ }^{\mathrm{b}} \mathrm{PS}=$ polystyrene, $\mathrm{PANI}=$ polyaniline.

${ }^{\mathrm{c}}$ Abbreviation samples name correspond to Tables 1,2.

${ }^{\mathrm{d}} \mathrm{DMSO}=$ dimethyl sulfoxide.

${ }^{\mathrm{e}} \mathrm{NMP}=\mathrm{N}$-methyl-2-pyrrolidone.

${ }^{\mathrm{f}}$ Tetrahydrofuran.

TABLE 5 Molecular weights of polymers ${ }^{\mathrm{a}}$.

\begin{tabular}{lcccc}
\hline Polymer & $\begin{array}{c}M_{\mathrm{w}}{ }^{\mathrm{b}} \\
(\mathrm{g} / \mathrm{mol})\end{array}$ & $\begin{array}{c}M_{\mathrm{n}}{ }^{\mathrm{c}} \\
(\mathrm{g} / \mathrm{mol})\end{array}$ & $\begin{array}{c}M_{\mathrm{v}}{ }^{\mathrm{d}} \\
(\mathrm{g} / \mathrm{mol})\end{array}$ & $M_{\mathrm{w}} / M_{\mathrm{n}}$ \\
\hline PS & $2.7 \times 10^{5}$ & $1.6 \times 10^{5}$ & $2.7 \times 10^{5}$ & 1.7 \\
PS-PANI3/ES1 & $3.9 \times 10^{5}$ & $2.3 \times 10^{5}$ & $3.9 \times 10^{5}$ & 1.7 \\
PS-PANI5/ES1 & $4.8 \times 10^{5}$ & $3.1 \times 10^{5}$ & $4.8 \times 10^{5}$ & 1.6 \\
PS-PANI10/ES1 & $3.3 \times 10^{5}$ & $1.4 \times 10^{5}$ & $3.3 \times 10^{5}$ & 2.4 \\
PS-PANI-D/ES1 & $3.7 \times 10^{5}$ & $2.6 \times 10^{5}$ & $3.6 \times 10^{5}$ & 1.5 \\
PS-PANI3-2/ES1 & $2.7 \times 10^{5}$ & $1.6 \times 10^{5}$ & $2.7 \times 10^{5}$ & 1.7 \\
PS-PANI5/ES2 & $2.4 \times 10^{5}$ & $1.5 \times 10^{5}$ & $2.4 \times 10^{5}$ & 1.7 \\
PS- PANI15/ES2 & $1.7 \times 10^{5}$ & $9.9 \times 10^{4}$ & $1.7 \times 10^{5}$ & 1.7 \\
P(St-co-ACN)- & $5.4 \times 10^{5}$ & $3.3 \times 10^{5}$ & $5.4 \times 10^{5}$ & 1.6 \\
PANI/ES1 & & & & \\
\hline
\end{tabular}

\footnotetext{
${ }^{\mathrm{a}}$ Abbreviation sample names correspond to Tables 1,2.

${ }^{b}$ Number-average molecular weight relative to polystyrene standard.

${ }^{c}$ Weight-average molecular weight relative to polystyrene standard.

${ }^{\mathrm{d}}$ Viscosity-average molecular weight relative to polystyrene standard.
} 
UV-vis absorption spectra of the polymers in NMP solution are shown in Figure 4. PANI (EB) and PS-PANI samples show two characteristic absorption bands in the magnification spectra (Figure 4, bottom). The quite weak absorption band at 300-400 $\mathrm{nm}$ of the resultant polymers is derived from $\pi-\pi^{*}$ transition of the benzenoid structure of PANI. ${ }^{17}$ The absorption band at 500$600 \mathrm{~nm}$ is derived from $\pi-\pi^{*}$ transition of quinoid structure of PANI. ${ }^{18}$ These absorption bands are typical of PANI emeraldine base. PS-PANI samples show these absorptions because of existence of small amount of PANI in the terminal of the PS.

TABLE 6 Spin concentration (radical conc.) of polymers.

\begin{tabular}{lc}
\hline \multicolumn{1}{c}{ Sample } & $C(\mathrm{spin} / \mathrm{g})^{\mathrm{a}}$ \\
\hline PANI $^{\mathrm{a}}$ & $2.4 \times 10^{18}$ \\
PS-PANI3/ES1 $^{\mathrm{a}}$ & $4.3 \times 10^{17}$ \\
PS-PANI5/ES1 $^{\mathrm{b}}$ & $2.1 \times 10^{17}$ \\
PS-PANI10/ES1 $^{\mathrm{b}}$ & $3.6 \times 10^{17}$ \\
PS $^{\mathrm{b}}$ & 0 \\
\hline
\end{tabular}

${ }^{\mathrm{a}}$ Abbreviation name corresponds to Tables 1,2.

${ }^{\mathrm{b}} \mathrm{Sp}$ in concentration evaluated with the ESR.

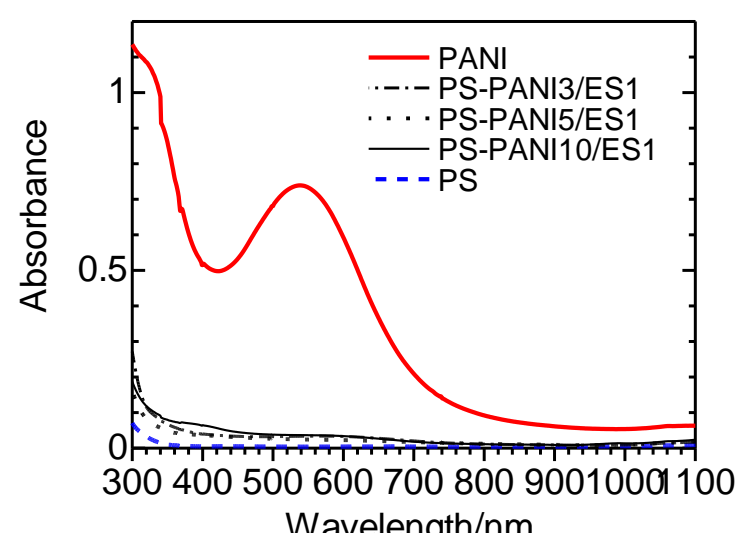

Radical detection of the samples is carried out with electron spin resonance (ESR) spectroscopy measurements in X-band, Table 6. Polarons in the PANI are detected with the ESR, although the reference PS shows no signals in the ESR. Spin concentration of PS-PANI is lower than that of PANI, indicating an existence of trace amount of polarons on the PANI site of PS-
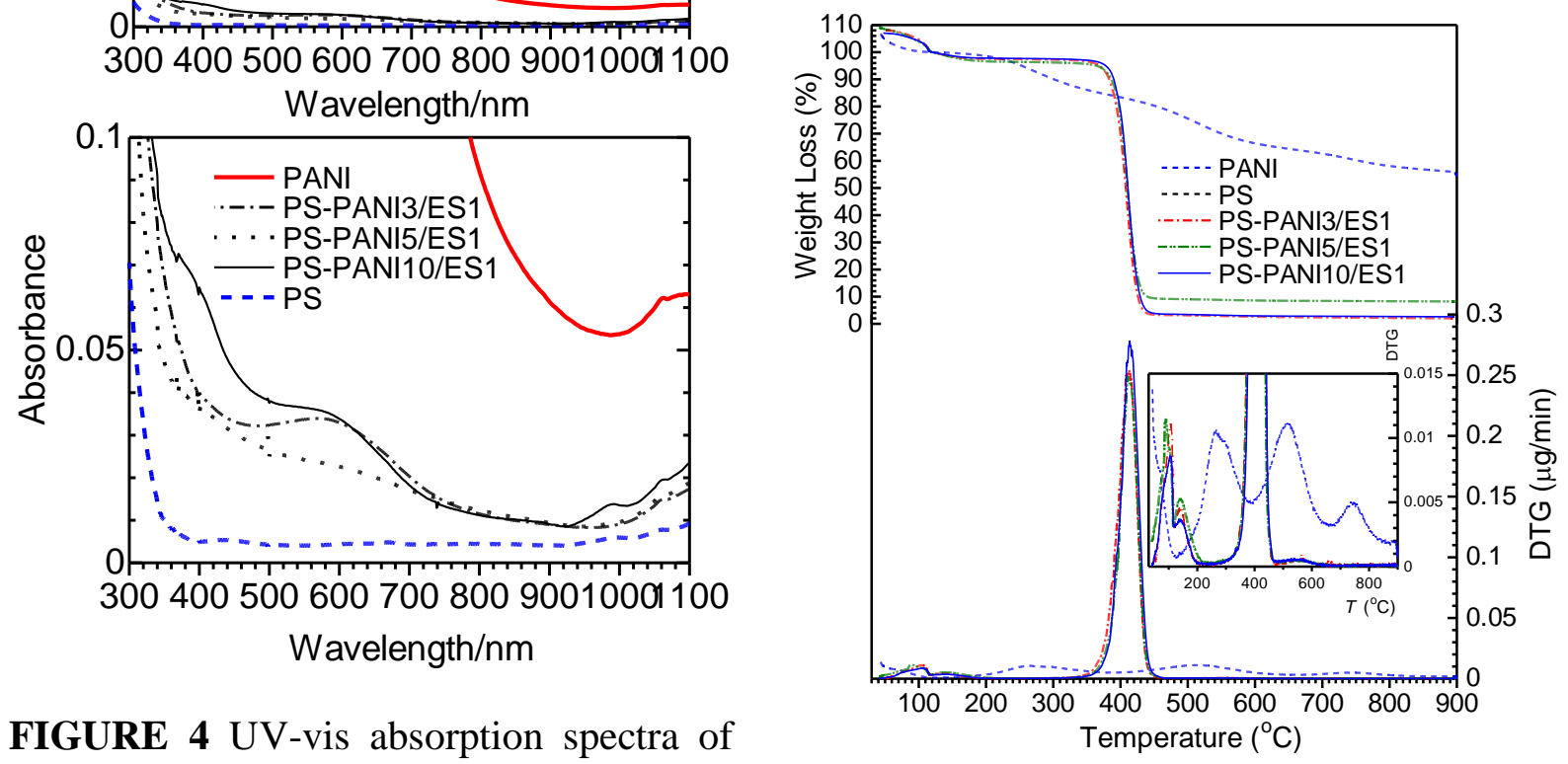

FIGURE 4 UV-vis absorption spectra of polyaniline (PANI) and polystyrene (PS) in $\mathrm{N}$-methyl-2-pyrrolidone (NMP) (top) and magnification of UV-vis spectra (bottom).

FIGURE 5 Thermal gravimetric (TG) and differential thermal analysis (DTG) curves of polymers. 
PANI after polymerization. Thermal properties of the polymers are examined with thermogravimetry (TG) and differential thermogravimetry (DTG) under argon flow, from room temperature to $900{ }^{\circ} \mathrm{C}$, Figure 5. Inset of Figure 5 shows magnification of the DTG curves. Weights of PS and PS-PANIs slightly decrease up to $400{ }^{\circ} \mathrm{C}$. The weight of PS and PS-PANIs drastically decrease at around $410{ }^{\circ} \mathrm{C}$, indicating thermal decomposition of PS. PS-PANIs show thermal behavior similar to that of PS. In Figure 5(bottom), PS have maxima in the DTG at around $140{ }^{\circ} \mathrm{C}$ and $180{ }^{\circ} \mathrm{C}$. These two signals $\left(<400{ }^{\circ} \mathrm{C}\right)$ may be due to surface oxidation. ${ }^{19}$ DTG curves of PANI show maxima at around $270{ }^{\circ} \mathrm{C}$ and $520{ }^{\circ} \mathrm{C}$ due to thermal decomposition of PANI, Figure 5 (bottom) ${ }^{20,21}$. However, PS-PANIs exhibit no detectable signals due to PANI in the range of $<400{ }^{\circ} \mathrm{C}$ and show small signal at $520{ }^{\circ} \mathrm{C}$, indicating the polymers contains very small amount of PANI component in the entire system.

\section{CONCLUSIONS}

Radical polymerizations were successfully performed with polarons at N position of PANI. This result demonstrates that charge carriers of conducting polymers for electrical conduction can function as a radical polymerization initiator. At the present stage, living radical polymerization using polarons is not achieved. However, this example provides a new possibility in both research fields of conducting polymer and plastic technologies.

\section{ACKNOWLEDGMENTS}

We would like to thank the OPEN FACILITY, Research Facility Center for Science and Technology, University of Tsukuba, for supporting measurement.

\section{REFERENCES}

1. Fox Jr, T. G., Flory, P. J., J. Am. Chem. Soc., 1948, 70(7), 2384-2395.

2. Fox, T. G., Flory, P. J., J. Polym. Sci., 1954, 14(75), 315-319.

3. Spencer, R. S., Dillon, R. E., J. Colloid Sci., 1949, 4(3), 241-255.

4. Chiang, J. C., MacDiarmid, A. G., Synth. Met., 1986, 13(1-3), 193-205.

5. MacDiarmid, A. G., Chiang, J. C., Richter, A. F., Epstein, A. A, Synth. Met., 1987, 18(1-3), 285-290.

6. Stafström, S., Bredas, J. L., Epstein, A. J., Woo, H. S., Tanner, D. B., Huang, W. S., MacDiarmid, A. G., Phys. Rev. Lett., 1987, 59(13), 1464.

7. MacDiarmid, A. G., Epstein, A. J., Synth. Met., 1995, 69.1, 85-92.

8. Rice-Evans, C. A., Nicholas J. Miller, George Paganga, Free Radic. Biol. Med. 1996, 20.7, 933-956.

9. Tang, J., Jing, X., Wang, B., Wang, F., Synth. Met., 1988, 24(3), 231-238.

10. Hwang, G. W., Wu, K. Y., Hua, M. Y., Lee, H. T., Chen, S. A., Synth. Met., 1998, 92(1), 39-46.

11. Cataldo, F., Maltese, P. Euro. Polym. J., 2002, 38(9), 1791-1803.

12. Bissessur, R., White, W. Mater. Chem. Phys., 2006, 99(2), 214-219.

13. Lee, Y. M., Kim, J. H., Kang, J. S., Ha, S. Y., Macromolecules, 2000, 33(20), 7431-7439.

14. Wei, Y., Hariharan, R., Patel, S. A. Macromolecules, 1990, 23(3), 758-764.

15. Stejskal, J., Trchová, M., Prokeš, J., Sapurina, I. Chem. Mater., 2001, 13(11), 4083-4086. 
16. Kakida, H., Tashiro, K., Kobayashi, M., Polym. J., 1996, 28(1), 30-34.

17. Pruneanu, S., Veress, E., Marian, I., Oniciu, L., J. Mat. Sci., 1999, 34(11), 2733-2739.

18. Weith, A. J., Turkington, V. H., Allen Jr, I., Ind. Eng. Chem., 1940, 32(10), 1301-1304.

19. Zheng, W. Y., Levon, K., Laakso, J., Oesterholm, J. E., Macromolecules, 1994, 27(26), 7754-7768.

20. Kaitsuka, Y., Hayashi, N., Shimokawa, T., Togawa, E., Goto, H. Polym., 2016, 8(2), 40.

21. Traore, M. K., Stevenson, W. T. K., McCormick, B. J., Dorey, R. C., Wen, S., Meyers, D. Synth. Met., 1991, 40(2), 137-153. 
Supporting Information for:

\title{
Polarons radical polymerization
}

\author{
Kohei Yamabe, Kuniharu Nakajima, Hiromasa Goto* \\ Division of Materials Science, Faculty of Pure and Applied Sciences, \\ University of Tsukuba, Tsukuba, Ibaraki 305-8577, Japan \\ *E-mail:gotoh@ims.tsukuba.ac.jp
}

Preparation of polyaniline emeraldine salt. Aniline was purchased from Wako Chemical, Japan, and purified by distillation. Sulfuric acid was purchased from Wako Chemical, Japan, and used as received. Ammonium persulfate (APS, $\left(\mathrm{NH}_{4}\right)_{2} \mathrm{~S}_{2} \mathrm{O}_{8}$ ) were purchased from Kanto Chemical, Japan, and used as received.

Distilled aniline and sulfuric acid $\left(\mathrm{H}_{2} \mathrm{SO}_{4}\right)$ were added to distilled water in a vial. The solution was stirred and cooled to $0{ }^{\circ} \mathrm{C}$ in an ice bath. APS in distilled water was added slowly to the vial to initiate oxidative polymerization. After $24 \mathrm{~h}$, the solution changed dark color and it was washed with a large volume of distilled water and methanol for removing oligomer and APS. The precipitate was filtered and dried in a vacuum to obtain PANI emeraldine salt ${ }^{\mathrm{S} 1}$. 

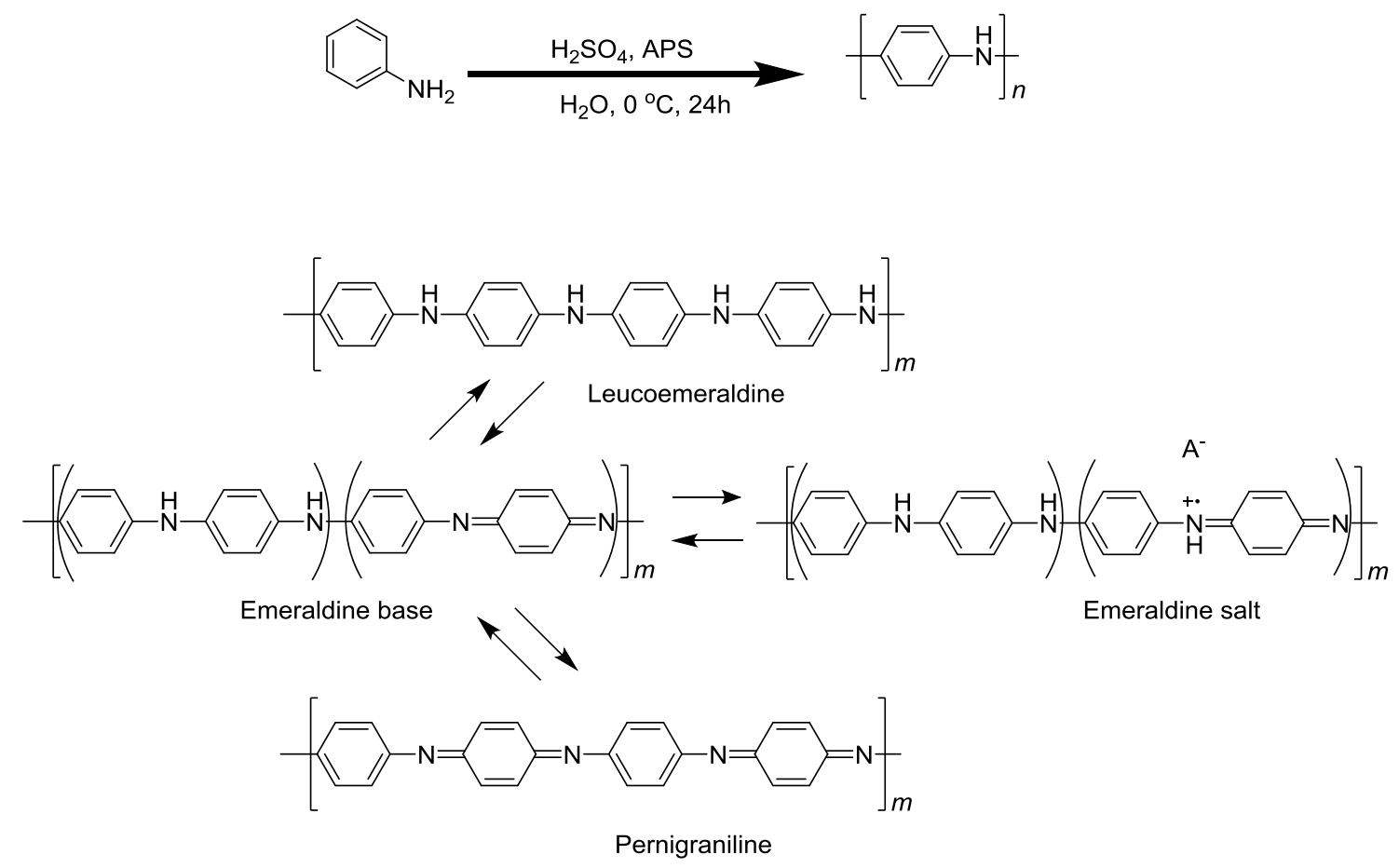

Scheme S1. Polymerization and chemical structure of PANI.

Characterization of polyaniline emeraldine salt. IR absorption of the PANI: FTIR $\left(\mathrm{KBr}, \mathrm{cm}^{-}\right.$ ${ }^{1}$ ): 1060, 1115 (C-H in-plane. on 1,4-ring and 1,2,4-ring); 1240 (B-B-B str.); 1300 (B-B-Q str.); 1485 (N-B-N str.); 1560 ( $\mathrm{N}=\mathrm{Q}=\mathrm{N}$ str.); $3380\left(\mathrm{NH}_{2}\right.$ sym. str., $\mathrm{NH}$ str.)

Polymerization by polarons in various temperature. PS were prepared with the same method except for reaction temperature condition (Table S1). PANI and styrene were dissolved in N-methyl-2-pyrrolidine (NMP) under an argon atmosphere. The reaction mixture was heated at room temperature or $140{ }^{\circ} \mathrm{C}$. After polymerization, minimal amount of tetrahydrofuran (THF) was added to dissolve the polymer. The polymer solution in THF was dropped into large volume of methanol. A pale pink precipitant appeared in the solution. After wash and filtration, the solid was dried in vacuum. At room temperature, PS was not obtained with PANI. At $140{ }^{\circ} \mathrm{C}$, polymer was obtained. Molecular weights of the polymer prepared at $140{ }^{\circ} \mathrm{C}$ are lower than those of polymerization at $75{ }^{\circ} \mathrm{C}$, and greater value of $M_{\mathrm{w}} / M_{\mathrm{n}}$ (dispersion) was confirmed. Living polymerization such as 2,2,6,6-tetramethylpiperidine 1-oxyl (TEMPO) initiated polymerization at $140{ }^{\circ} \mathrm{C}$ could not be occurred by the PANI initiator. 
Table S1. Preparation of polystyrene at various temperatures.

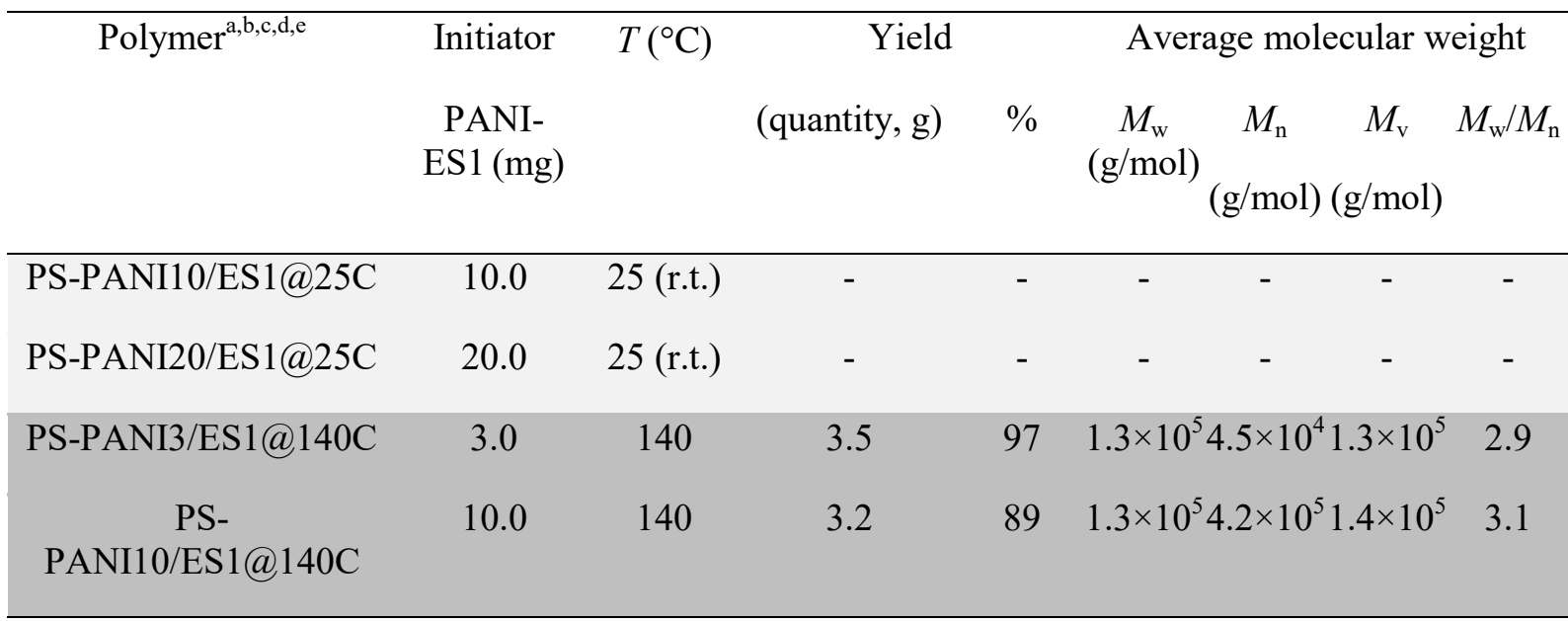

${ }^{\mathrm{a}}$ Mon. $=$ styrene, $4.0 \mathrm{~mL}$

${ }^{b} \mathrm{PANI}=$ polyaniline.

${ }^{\mathrm{c}} \mathrm{PANI}-\mathrm{ES} 1=$ polyaniline emeraldine salt form with spin conc. of $2.4 \times 10^{18} \mathrm{spin} / \mathrm{g}$.

${ }^{\mathrm{d}}$ Reaction time $=24 \mathrm{~h}$.

${ }^{\mathrm{e}}$ Polymerization solvent $=\mathrm{N}$-methyl-2-pyrrolidone $(\mathrm{NMP})$.

Evaluation method of qualitative solubility. Polymers (ca. $100 \mathrm{mg}$ ) were dissolved in a large amount of solvent $(30 \mathrm{~mL}$ ) by shaking test tube (in Figure S1). "++, well soluble" means that polymer was completely dissolved within $1 \mathrm{~h}$. "+, partially soluble" means that polymer was partially dissolved within $1 \mathrm{~h}$. "-, slightly soluble or insoluble" means that polymer was slightly or not dissolved within $1 \mathrm{~h}$.

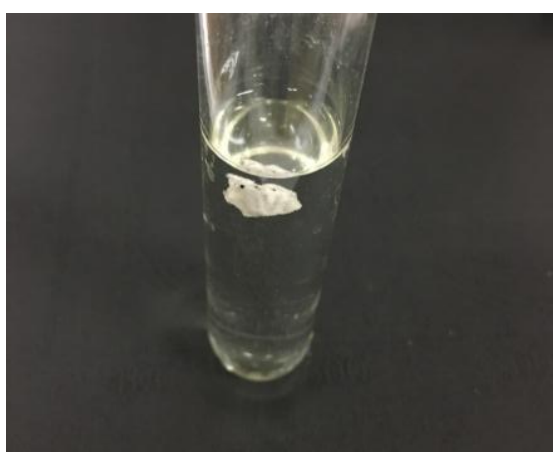

\section{Reference}

[S1] Cao, Y., Smith, P., Heeger, A. J. Synth. Met., 1989, 32(3), 263-281.

Figure S1. Solubility test in common organic solvent. 


\section{Abbreviations}

PANI $=$ Polyaniline.

\section{Polystyrene prepared with polarons of polyaniline emeraldine salt.}

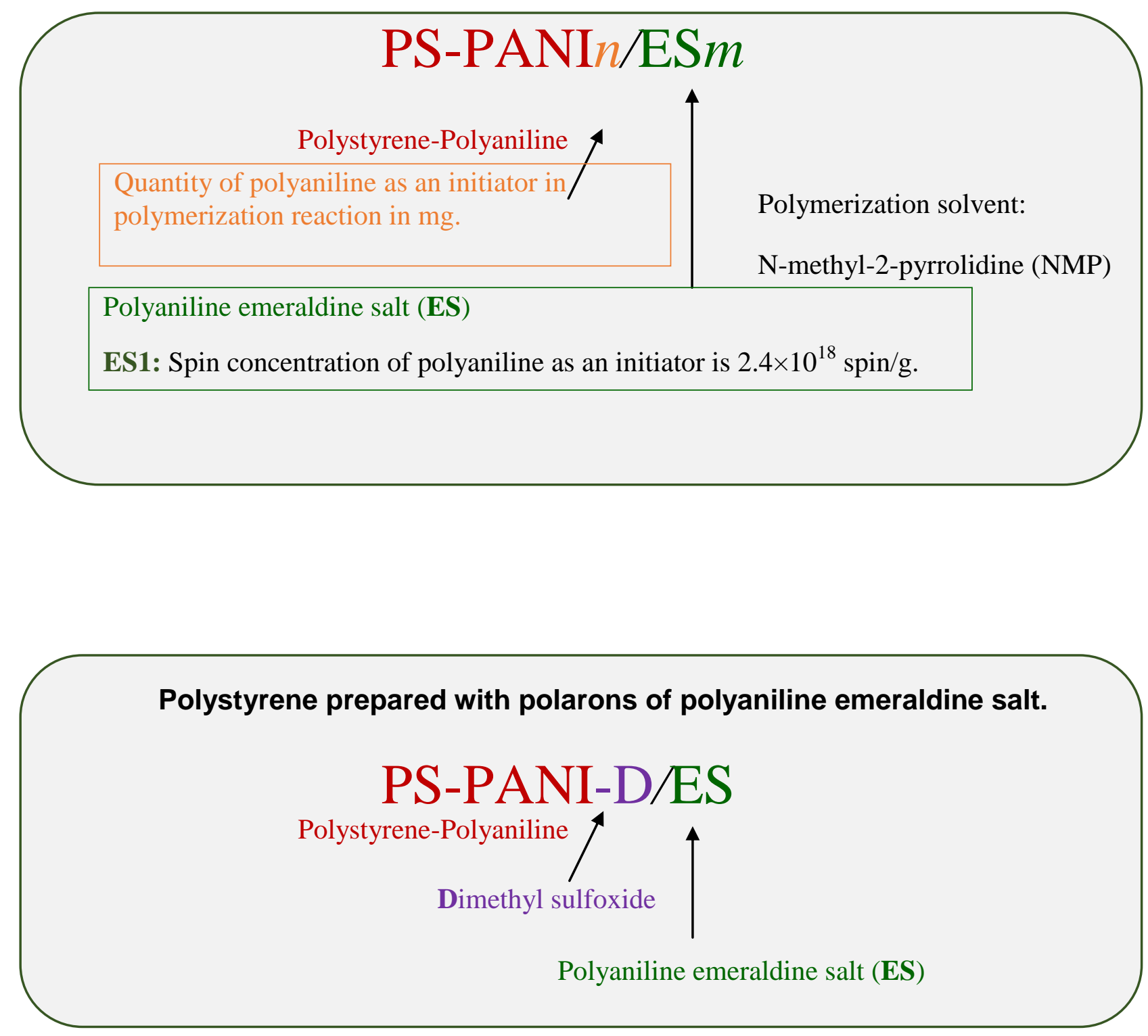


Polystyrene prepared with polarons of polyaniline emeraldine base.

\section{PS-PANI $n / E B$}

Polystyrene-Polyaniline $\$

Quantity of polyaniline as an

initiator in polymerization reaction

in $\mathrm{mg}$.
Polyaniline emeraldine base (EB) with spin concentration of $1.8 \times 10^{15} \mathrm{spin} / \mathrm{g}$.

Copolymer of polystyrene and polyacrylonitrile prepared with polarons of polyaniline emeraldine salt.

\section{$\mathrm{P}(\mathrm{St}-\mathrm{co}-\mathrm{ACN}) / \mathrm{PANI} / \mathrm{ES}$}

Poly(styrene-co-acrylonitrile) Polyaniline emeraldine salt (ES) 